\title{
The Research of Bidding Model Based on the Generalized Order Statistics
}

\author{
Chaohe Song \\ 451 HuangShan Road \\ Hefei 230031, China
}

Tel: 86-551-576-1233 E-mail: lisong828-2006@163.com

\begin{abstract}
In the Seller Bidding of order statistics bidding function, the expected deviation of the last non-winner's price and the winner's price is a decrease function of the number of bidder. This paper analysis and know that the expected deviation of the last non-winner's price and the winner's price decrease with the increasing of the number of bidder on the Seller Bidding of the generalized order statistics.
\end{abstract}

Keywords: The Generalized Order Statistics, Bidding Price Function, Seller Bidding

\section{Reviews}

Anand Paul and Genaro Gutierrez prove that the expected returns of bidding proprietor in a seller's bidding is a monotonically decreasing function of the number of bidders if the population from which the valuations are sampled is characterized by a concave distribution function. BUIOW and Klemperer prove that expected revenue with $\mathrm{N}+1$ bidders exceeds expected revenue with $N$ bidders if the bidders' valuations are either independent. Now we study bidding model in the Generalized Order Statistics and analysis the relations between the number of bidder with the expected the expected deviation of the last non-winner's price and the winner's price.

\section{Definition}

The concept of generalized order statistics (gos) was given by Kamps (1995) as below (see (HASEEB, 2004)):

Let $F(\tilde{X})$ be an absolutely continuous distribution function $(d f)$ with probability density function $(p d f) \mathrm{f}(\tilde{X})$, Let $n \in N, n \geq 2, k>0, \tilde{m}=\left(m_{1}, m_{2}, \cdots, m_{n-1}\right) \in R^{n-1}, M_{i}=\sum_{j=i}^{n-1} m_{j}$, such that $\gamma_{i}=k+n-i+M_{i}>0$ for all $i \in\{1,2, \cdots, n-1\}$. Then $X(i, n, \tilde{m}, k), i=1,2, \cdots, n$ are called generalized order statistics (gos) if their joint probability density function is given by

$$
k\left(\prod_{j=1}^{n-1} \gamma_{j}\right) \prod_{i=1}^{n-1}\left[1-F\left(x_{i}\right)\right]^{k-1} f\left(x_{i}\right)\left[1-F\left(x_{n}\right)\right]^{k-1} f\left(x_{n}\right)
$$

on the cone $F^{-1}(0+)<x_{1} \leq x_{2} \leq \cdots \leq x_{n}<F^{-1}(1)$ of $R^{n}$

For $m_{1}=m_{2}=\cdots=m_{n-1}=m$, the $g o s$ will be denoted as $X(i, n, m, k)$ and its $p d f$ is given by Kamps (1995) as:

$$
f_{X(i, n, m, k)}(x)=\frac{c_{i-1}}{(i-1) !}[1-F(x)]^{\gamma_{i}-1} f(x) g_{m}^{i-1}(F(x))
$$

Where $C_{i-1}=\prod_{j=1}^{i} \gamma_{j}, \gamma_{j}=k+(n-j)(m+1) ; g_{m}(x)=\left\{\begin{array}{cc}\frac{1-(1-x)^{m+1}}{m+1} & m \neq-1 \\ \log \left(\frac{1}{1-x}\right) & m=-1\end{array} \quad x \in[0,1)\right.$ 


\section{Bidding Model Setup}

We first analyze a special bidding. Denote the $i$ th generalized order statistics in a sample of size $N$ by $\tilde{X}_{i: N}$ and suppose there are $N$ bidders competing to sell a good. Each bidder has a function valuation $\tilde{X}_{(i, N, m, k)}$. The buyer assumes that the function valuations of the bidders are iid random variables. Prices are bid in a descending sequence by individual bidders until only one bidder remains, the winner. The valuation of the winner is the first generalized order statistics.

$$
E\left(\tilde{X}_{K+1: N}-\tilde{X}_{K: N}\right)=C_{K}^{N} \int_{0}^{\infty} F(\tilde{X})^{K}(1-F(\tilde{X}))^{N-K} d \tilde{X}
$$

which is a standard result when the parent distribution is positive valued (see Udo Kamps, 1995).

The expectation of the deviation between the second and first generalized order statistic of a random sample of size $N$ in a seller's bidding by

$$
E\left(\tilde{X}_{2: N}-\tilde{X}_{1: N}\right)=N \int_{0}^{\infty} F(\tilde{X})\{1-F(\tilde{X})\}^{N-1} d \tilde{X}
$$

Now we denote the deviation between expected of $\mathrm{N}$ bidders and $N+1$ bidders by $D(N)$.

$$
\begin{aligned}
D(N) & =E(N)-E(N+1) \\
& =N \int_{0}^{\infty} F(\tilde{X})\{1-F(\tilde{X})\}^{N-1} d \tilde{X}-(N+1) \int_{0}^{\infty} F(\tilde{X})\{1-F(\tilde{X})\}^{N} d \tilde{X} \\
& =\int_{0}^{\infty} F(\tilde{X})\{1-F(\tilde{X})\}^{N-1}\{(N+1) F(\tilde{X})-1\} d \tilde{X}
\end{aligned}
$$

Note that as $N$ tends to infinity£this integral tends to zero by Lévesque's Dominated Convergence Theorem£We are, however, interested in the situation when $N$ is finite. In order to guarantee $D(N)>0$, we assume that the valuations are distributed on a compact interval $[a, b](0 \leq a<b \leq)$ on the positive real line and that the density function of the underlying random variable is continuous.

\section{Important Result}

Lemma 1: Let $m_{1}=m_{2}=\cdots=m_{i-1}=m$, the $d f$ of the $i$ th uniform gos is denoted by $\varphi_{i, N}(x)=$ $f_{U(i, N, m, k)}(x)=\frac{c_{i-1}}{(i-1) !}(1-x)^{\gamma_{i}-1} g_{m}^{i-1}(x), x \in(0,1), 1 \leq i \leq n$ (see(Anand Paul, 2003) and (Udo Kamps, 1995))

Lemma 2 Let $m_{1}=m_{2}=\cdots=m_{i-1}=m$ and $i \in\{1,2, \cdots, n\}$ fthen we have, $\phi_{i, N}(x)=1-C_{i-1}(1-x)^{k+N-i+M_{i}}+$ $\sum_{j=0}^{i-1} \frac{1}{j ! C_{i-j-1}} g_{m}^{j}(x), x \in(0,1)$ and $F_{X(i, N, m, k)}(x)=\phi_{i, N}(F(x))$ (see(CRAMLR,E, 2000)).

Lemma $3 \int_{a}^{b} F(\tilde{X})[1-F(\tilde{X})]^{N-1}[(N+1) F(\tilde{X})-1] d F(\tilde{X})>0$ for all function $F(\tilde{X})$ where $J(\tilde{X})=F(\tilde{X})\{1-$ $F(\tilde{X})\}^{N-1}\{(N+1) F(\tilde{X})-1\}$ is obvious.

The Stieltjes integral $\int_{a}^{b} J(\tilde{X}) d F(\tilde{X})$ in Lemma 3 can be rewritten as $\int_{a}^{b} J(\tilde{X}) f_{(i, N, m, k)}(x) d x$ where $f_{(i, N, m, k)}(x)$ is $p d f$ of the distribution of valuations

Theorem 1 The expected deviation of the last non-winner's price and the winner's price in a seller's bidding is a monotonically decreasing function of the number of bidders if the bidder from which the valuations are sampled is characterized by a concave distribution function in the Generalized Order Statistics.

In theorem 1, we only considerate two cases: case I $m_{1}=m_{2}=\cdots=m_{i-1}=m \neq-1$ and Case II $m_{1}=m_{2}=$ $\cdots=m_{i-1}=m=-1$.

For case I: From Lemma 1, we have $\varphi_{2, N}(x)=\frac{\gamma_{1} \gamma_{2}}{m+1}\left\{(1-x)^{\gamma_{2}-1}-(1-x)^{\gamma_{1}-1}\right\}$. So we know the probability density function and the distribution function of 2 nd generalized order statistics ( gos ) as follows:

$$
\begin{gathered}
f(c)=\varphi_{2, N}(c)=\frac{\gamma_{1} \gamma_{2}\left[(1-c)^{\gamma_{2}-1}-(1-c)^{\gamma_{1}-1}\right]}{(m+1)} \\
F(c)=\int_{a}^{c} \varphi_{2, N}(x) d x=\frac{\gamma_{1}(1-c)^{\gamma_{2}}-\gamma_{2}(1-c)^{\gamma_{1}}+\gamma_{2}(1-a)^{\gamma_{1}}-\gamma_{1}(1-a)^{\gamma_{2}}}{m+1}
\end{gathered}
$$


And from Definition and Lemma 2, we can rewrite the generalized order statistics distribution function $F_{X(i, N, m, k)(x)}$ and the probability density function $f_{X(i, N, m, k)}(x)$ :

$$
\begin{gathered}
F_{X(2, N, m, k)}(x)=\frac{\gamma_{2}[1-F(x)]^{\gamma_{1}}-\gamma_{1}[1-F(x)]^{\gamma_{2}}+1}{m+1} \\
f_{X(2, N, m, k)}(x)=\frac{\gamma_{1} \gamma_{2}}{m+1}\left\{[1-F(x)]^{\gamma_{2}-1}-[1-F(x)]^{\gamma_{1}-1}\right\} f(x)
\end{gathered}
$$

From Lemma 3 we know if $f_{X(2, N, m, k)}(x)$ were a decreasing function such that $f_{X(2, n, m, k)}(c)=1$ and $F_{X(2, n, m, k)}(c)=$ $1 /(N+1)$, then $J(\tilde{X})$ is 0 at $x=a, c$ and $b$, negative on the interval $(a, c)$ and positive on the interval $(c, b)$. we would have $\int_{a}^{b} J(\tilde{X})\left(1-f_{(i, N, m, k)}(x)\right) d x>0$ in $[a, c]$ and $[c, b]$, then we have integral unequal $\left.\int_{a}^{b} J(\tilde{X}) d x>\int_{a}^{b} J(\tilde{X}) f_{i} i, N, m, k\right)(x) d x>0$ and Theorem 1 would be proved.

But if $f_{X(2, n, m, k)}(c)=1$ is unknown here, So we reduce two equation through substituting (5) and (6) to

$$
\left\{\frac{N}{N+1}+k\left[\frac{v(b)-v(c)}{m+1}\right]^{k}\right\} \frac{(1-c)^{\gamma_{1}}-(1-c)^{\gamma_{2}}}{1-c}+\frac{(v(b)-v(c))}{\gamma_{1} \gamma_{2}}=0
$$

where $v(t)=\gamma_{1}(1-t)^{\gamma_{2}}-\gamma_{2}(1-t)^{\gamma_{1}}$. Let

$$
T_{1}(x)=\left\{\frac{N}{N+1}+k\left[\frac{v(b)-v(x)}{m+1}\right]^{k}\right\} \frac{(1-x)^{\gamma_{1}}-(1-x)^{\gamma_{2}}}{1-x}+\frac{(v(b)-v(x))}{\gamma_{1} \gamma_{2}}
$$

. Where we would have

$$
T_{1}(a)=\frac{-\gamma_{1}\left\lfloor(1-a)^{\gamma_{2}}-(1-a)^{\gamma_{1}}\right\rfloor}{(N+1)(1-a)}-1<0
$$

and $T_{1}(b)=\frac{N_{\gamma_{1}}\left\lfloor(1-b)^{\gamma_{2}}-(1-b)^{\gamma_{1}}\right\rfloor}{(N+1)(1-b)}>0$. So we have at least a point $c$ which make $T_{(c)}$ equal to 0 for $T_{1}(x)$ is a continuous function.

For Case II: From Lemma 1, we have $\varphi_{2, N}(x)=-\gamma_{1} \gamma_{2}(1-x)^{\gamma_{1}-1} \ln (1-x)$. So we obtain the density function and the distribution function of 2 nd generalized order statistics (gos) as follows:

$$
\begin{gathered}
f_{2, N}(c)=\varphi_{2, N}(c)=-\gamma_{1} \gamma_{2}(1-c)^{\gamma_{1}-1} \ln (1-c) \\
F_{2, N}(c)=\int_{a}^{c} \phi_{2, N}(x) d x=\frac{(1-c)^{\gamma_{1}}}{\gamma_{1}^{2}} \ln \frac{(1-c)^{\gamma_{1}}}{e}-\frac{(1-a)^{\gamma_{1}}}{\gamma_{1}^{2}} \ln \frac{(1-a)^{\gamma_{1}}}{e}
\end{gathered}
$$

From Definition and Lemma 2, the distribution function $F_{X(i, N, m, k)}$ and the probability density function $f_{X(i, N, m, k)}$ in generalized order statistics can be rewrote:

$$
\begin{gathered}
F_{X(z, N, m, k)}(x)=\int_{0}^{F(x)} \varphi_{i, N}(t) d t=[1-F(x)]^{k}\{k \ln [1-F(x)]-1\}+1 \\
f_{X(2, N, m, k)}(x)=-k^{2}[1-F(x)]^{k-1} \ln [1-F(x)] f(x)
\end{gathered}
$$

As case I, we analysis Case II and substitute (7) and (8) to above two equation and reduce them to

$$
k\left\{\frac{N}{N+1}-[u(b)-u(c)]^{k}\right\}(1-c)^{\gamma_{1}-1} \ln (1-c)+\frac{u(b)-u(c)}{\gamma_{1} \gamma_{2}}=0
$$

where

$$
u(t)=\frac{(1-t)^{\gamma_{1}}}{\gamma_{1}^{2}} \ln \frac{(1-t)^{\gamma^{1}}}{e}
$$

Let

$$
T_{2}(x)=k\left\{\frac{N}{N+1}-[u(b)-u(x)]^{k}\right\}(1-x)^{\gamma_{1}-1} \ln (1-x)+\frac{u(b)-u(x)}{\gamma_{1} \gamma_{2}} .
$$


Then we would have

$$
T_{2}(a)=\frac{k(1-a)^{\gamma_{1}}}{(N+1)(1-a)} \ln (1-a)-1<0
$$

and

$$
T_{2}(b)=\frac{k N(1-b)^{\gamma_{1}}\left[1-(1-b)^{m+1}\right]}{(N+1)(1-b)}>0
$$

So we have at least a point $c$ which make $T_{2}(c)$ equal to 0 for $T_{2}(x)$ is a continuous function also.

From case I and Case II, we would have $\int_{a}^{b} J(\tilde{X}) d x>\int_{a}^{b} J(\tilde{X}) f_{(r, n, m, k)}(x) d x>0$ if $m_{1}=m_{2}=\cdots=m_{i-1}=m$. So the result has been proved.

\section{References}

Anand Paul and Genaro Gutierrez, (2003), Mean sample spacing, sample size and variability in an biddingtheoretic frameworkfOperations Research Letters, 32, 103-108.

CRAMLR, E and KAMPS, U, (2000), Relations for expectations of functions of generalized order statistics£Journal of Statistical Planning and Inference, 89, 79-89.

HASEEB ATHAR-HASAN MATEEN-UL ISLAM, (2004), Recurrence relations for single and product moments of generalized order statistics from a general class of distribution, vol. LXII, n.3, 327-337.

H. David, Order Statistics, New York: Wiley, (1981).

J. Bulow, P. Klemperer, (1996), Bidding versus negotiations, America Economic, Rev 86, 180-194.

KAMPS. U. and CRAMFR. E. (2001), On distribution of generalized order statistics, Statistics, 35, 269-280.

M. Shaked, J. G. Shanthikumar, Stochastic Order And Their Application, Academic Press, New York, 1994.

Udo Kamps, (1995), A concept of generalized order statistics, Journal of Statistical Planning and Inference, $48,1-23$. 